\title{
Alfabetización, transformación y conciencia social. Sistematización de una experiencia
}

\author{
Luz Mery Guevara ${ }^{1}$
}

\begin{abstract}
Resumen
Objetivo. Analizar y sistematizar la experiencia de un Programa de Educación Integral para jóvenes y adultos, desarrollado por la Vicerrectoría de Desarrollo Regional y Participación Comunitaria de la Universidad Nacional Abierta y a Distancia UNAD y el Consejo Noruego para refugiados, en el periodo comprendido entre 200082010 .
\end{abstract}

Método. Se utilizaron talleres, entrevistas, conversatorios y revisión documental y contó con la participación de alfabetizadores, alfabetizados, coordinadores pedagógicos, coordinadores institucionales, comunidades locales y líderes locales.

Resultados. Se analiza la experiencia en cuanto alcances, apuestas, avances y limitantes para consolidar el Modelo de Alfabetización de la UNAD.

Palabras clave: Alfabetización, desplazamiento, solidaridad, derechos, pedagogía popular. 


\title{
Literacy transformation and social awareness. Experience Systematization
}

\begin{abstract}
Objective. Analyze and systematize the experience of a comprehensive education program for youth and adults, developed by the Vice Presidency of Regional Development and Public Participation of UNAD and the Norwegian Refugee Council in the period 200082010 .
\end{abstract}

Method. Workshops, interviews, discussions and document review were used and included the participation of educators, students, educational coordinators, institutional coordinators, local communities and local leaders.

Results. Experience regarding scope, predictions, progress and constraints to consolidate UNAD Model Education are analyzed.

Keywords: Literacy, displacement, solidarity, rights, popular education.

\section{Una experiencia y el aprendizaje ${ }^{2}$}

Cada vez que llega un tiempo nuevo el que no es la mera prolongación del pasado sino portador de un mundo nuevo o de cambios radicales y masivos en el que está en curso, nada vuelve a significar lo mismo

Aníbal Quijano, 2009

La Universidad Nacional Abierta y a Distancia UNAD, es un ente universitario, autónomo de carácter nacional, con régimen especial y dependiente del Ministerio de Educación Nacional. Parte de su misión centra su esfuerzo en ofrecer educación para todos a través de la investigación, la acción pedagógica y la proyección social, entre otras. Para lograr este propósito la Vicerrectoría de 
Desarrollo Regional y Proyección Comunitaria ofrece el Programa de Educación Básica para la Inclusión Social EBIS y como parte de este, el de alfabetización. El programa EBIS entiende la alfabetización como un dispositivo de inclusión social, y por lo tanto, permite que los sujetos se comprendan como ciudadanos desde una perspectiva de derechos y convivencia. El proceso formativo permite que las personas involucradas desarrollen la capacidad de interpretar la realidad para transformarla, haciendo uso creativo de los conocimientos, valores y habilidades a partir de la formación académica y la apropiación de saberes culturales propios de las diferentes comunidades que participan (UNAD, 2010).

El Consejo Noruego para Refugiados, NRC, por su parte considera que su rol dentro del contexto colombiano actual, es apoyar a las instituciones del Estado y la sociedad civil colombianas, en la búsqueda de alternativas sostenibles para la protección y la promoción de los derechos fundamentales (políticos, económicos y sociales), de las víctimas del conflicto armado, con especial énfasis en las poblaciones desplazadas por el conflicto y en la perspectiva de apoyar la construcción de soluciones duraderas a su situación. Para el Consejo es claro que la educación convencional, es limitada en su respuesta a las poblaciones víctimas del conflicto y que su aporte a la construcción de soluciones duraderas a los problemas humanitarios por los que pasa Colombia, implica el apoyo al desarrollo de alternativas educativas, especialmente focalizadas en las víctimas y adecuadas a sus circunstancias socio-culturales, socio-afectivas y socioeconómicas, lo que significa generar modelos educativos pertinentes, incluyentes y de calidad.

En este contexto inició el convenio entre la UNAD y el NRC con el fin de desarrollar el programa de Educación Integral para jóvenes y adultos con presencia en diferentes regiones del territorio nacional colombiano, desde el año 2008. Este programa estableció el Ciclo I y el Ciclo II, con miras al logro de su objetivo. Con el propósito de revisar su ejercicio, esta propuesta recoge el interés de la UNAD, concretamente de la Vicerrectoría de Desarrollo Regional VIDER y el Consejo Noruego para Refugiados NRC, para reflexionar sobre sus alcances, apuestas, avances y limitantes. La pretensión además surgió de la necesidad de diseñar algunas herramientas estratégicas del programa Educación Integral de Adultos, a partir del trabajo en curso particularmente para los municipios de Santa Marta, Cúcuta, Fundación y Soacha. Para las dos instituciones: VIDER y NRC, el proyecto Educación Integral de Adultos, había tenido importantes avances y aprendizajes en su pilotaje en el terreno, sin embargo, no se había logrado producir un conjunto sistémico de los instrumentos constitutivos del modelo para su implementación y su futura expansión en el ambito nacional (UNAD, 2010).

El ejercicio propuesto cobró sentido, en la medida en que para el modelo pedagógico y para las partes, fue de suma importancia, desarrollar los productos que respondieran a algunas de las preocupaciones en las que se incluían: No 
contar con un documento sintético de presentación del "modelo" en: a) su enfoque pedagógico, b) su propuesta de institucionalización y c) el sistema de operación y gestión. Este documento se consideraba básico para la validación del modelo por el Ministerio de Educación, así como para la promoción, divulgación, convocatoria y concertación con los grupos y sectores interesados en el modelo: comunidades, secretarías de educación, agencias de cooperación internacional, entre otros.

De igual forma, se constituía en preocupación para las partes que a pesar de haberse diseñado con un conjunto de documentos que podrían constituir la Caja de herramientas del facilitador, estos eran "asistémicos" y los instrumentos necesitaban ser ajustados e integrados, de tal manera que se constituyeran en un todo orgánico: Se mencionaban entre otros: a) el instrumento de caracterización comunitaria; b) el documento de competencias para desarrollar en los participantes, c) la metodología por proyectos pedagógicos, d) la relación unidad de tiempo- tema-dinámica de grupo-, e) la ruta didáctica, f) la cartilla inconclusa y los materiales de trabajo de los participantes.

Finalmente, era necesario elaborar un documento suficientemente comunicativo y didáctico para presentar los procesos de formación de los facilitadores, (aquí se referenciaba el diplomado), desde el enfoque, curricular, la metodología hasta el enfoque pedagógico, con una clara definición de su pertinencia, contextualización y uso de materiales. Esta semblanza permitió plantear un interrogante común acerca de ¿Cuáles eran los avances, problemáticas, dinámicas y perspectivas del programa Educación Integral para Jóvenes y Adultos hasta la fecha, en el marco del convenio UNAD-NRC?

Se determinó de común acuerdo entre las partes, realizar un proceso que diera cuenta de estas condiciones y para ello, se definieron tres sectores para realizar dicho ejercicio: Soacha, Santa Marta y Cúcuta. Se consideró necesario el desplazamiento hasta los sitios, para conversar con los actores y participantes y observar el desarrollo de los procesos, además de la revisión de los documentos que orientaban la experiencia. Entre los documentos se incluyeron: Manual del Tutor, Secuencia Didáctica, Proyectos de Investigación Pedagógica, esquemas didácticos, diarios de campo, planeadores, cartilla inconclusa, entre otros.

Con base en la definición de las categorías se constituyeron algunos referentes para la conceptualización final que dan sentido y soporte a la experiencia, especialmente la educación popular, en tanto se configura como una apuesta de educación para todos y todas y para toda la vida; en este sentido entendimos que es la configuración de un sujeto autónomo y responsable comprometido con la transformación social. De igual manera recogimos los supuestos de ella en tanto opción para hacer frente a las condiciones de una política neoliberal que avasalla mentes y cuerpos y priva a las poblaciones en condición de vulnerabilidad del goce de un derecho fundamental como es la educación y el acceso al 
conocimiento. De otro lado y como una de las prácticas más significativas de la educación popular, la alfabetización se constituye en un aprendizaje que le permite a los sujetos aprender a escribir su vida, como testigo de su historia; así pues el método de alfabetización es asumido por Freire como práctica de la libertad; en este sentido, la pedagogía se hace antropología y el método no apunta a la eficiencia técnico-pedagógica, sino a la capacidad de potenciar la población para transformar la realidad.

Para nuestro propósito y en coherencia con nuestra apuesta epistemológica definimos la sistematización como la ruta para dar cuenta de la experiencia, asumiéndola como una práctica investigativa del orden cualitativo en el ámbito social y educativo, y reconociendo su aporte además, como proceso de evaluación de experiencias en donde la dicotomía sujeto que conoce y sujeto conocido desaparece y el interés por contrastar la teoría y la práctica se fortalecen, a partir de la narración y reconocimiento de los actores implicados y su aprendizaje a través de ella.

\section{Algunos referentes conceptuales y teóricos}

La educación popular que comprende la educación formal y permanente, la no formal y ocasional, existentes en una sociedad multicultural y los enfoque prácticos y teóricos, se configura en una apuesta que centra sus objetivos en una educación para toda la vida; en construir y desarrollar autonomía y sentido de responsabilidad; reforzar las capacidades para transformar la economía, cultura y sociedad; promover la coexistencia, tolerancia y la participación activa, conciente y creativa de los ciudadanos y ciudadanas. Como resultado de esto, el sujeto se autoconfigura, se descubre y se conquista reflexivamente como sujeto de su propio destino histórico (CEAAL, 1997). Tiene claramente implicaciones en el ámbito de lo educativo que se relacionan con la necesidad del diálogo, la preocupación por desarrollar capacidades, actitudes y conocimientos que permitan el pleno ejercicio de una ciudadanía que rescate la participación y conquiste y defienda sus derechos.

La hegemonía neoliberal, economía de mercado, democracia política y liberalismo social resumen el proyecto mundial que todos los países deben aspirar. La eficiencia, la acumulación de riqueza, la competencia, la conquista de personas y mercados, la capacidad de disuasión aparecen como la máxima expresión de los valores fundantes del modelo civilizatorio que se nos impone. El poder adquiere su principal fuente de sostenimiento, en el uso del conocimiento y de la información. La creación y apropiación tecnológica y científica son los ejes del desarrollo económico y de la conquista de los mercados. Esta sociedad del conocimiento que promete el paraíso a los inteligentes y esforzados otorga a las universidades del "primer mundo", el lugar de casas de la excelencia y por 
ello casas exclusivas. Se han creado empresas internacionales de "cazadores de talentos" para alimentar a las grandes compañías transnacionales y ahora a los gobiernos. Los medios de comunicación se afirman como un instrumento de carácter estratégico para el vínculo con las sociedades (Ramírez, 2010).

Para hacer frente a esta problemática de tendencia avasallante, surge la educación popular como propuesta político-pedagógica comprometida con una transformación educativa que involucre, la participación activa de los sujetos en sus propios aprendizajes y en su implicación cualitativa, sobre sus procesos de vida personal y colectiva. Recoge los espacios comunitarios y populares como oportunidad para abrir múltiples posibilidades de reflexión, producción, re-elaboración y orientación de procesos de intervención educativa y social, en ámbitos como la pedagogía, el desarrollo social, la cultura, la política, la comunicación, la educación y las nuevas exclusiones sociales.

Tenemos entonces a manera de precisiones que algunas de las características de la educación popular se reconocen en la posibilidad de hacer una lectura crítica y civilizadora de la estructura, de las condiciones de exclusión, desigualdad y marginalidad de las sociedades latinoamericanas que privan al grueso de la población, a ser, tener, hacer, vivir, manteniéndolas lejos del poder y la posibilidad de acción; esta lectura se constituye en su intención éticopolítica de carácter transformador que orientan las prácticas de esta educación. Esta ética se compromete con los excluidos, la alteridad, los nuevos actores sociales, la diversidad, los derechos humanos, la ciudadanía, la igualdad, la solidaridad, la autorrealización, la felicidad, entre otros. Así mismo se asume una intencionalidad ético-pedagógica de carácter emancipatorio que se traduce en la oportunidad de transformar las realidades sociales y sus relaciones de dominación en la vía de construir un nuevo orden social más justo y democrático. En este sentido, pretende democratizar el conocimiento y cambiar las relaciones de poder entre quienes conocen y los que no. Reconoce un sentido de poder y colonialidad del pensamiento hegemónico fortalecido en la práctica educativa y pedagógica tradicional y formal que mantiene y perpetúa estas relaciones, con claros efectos en el saber y el hacer; por tanto, sostiene que es a partir del conocimiento de la realidad y su análisis crítico, como se puede dar la acción liberadora y transformadora de estas condiciones.

Otra característica de la educación popular es que pretende reconstruir al sujeto social, en cuanto sujeto popular e histórico que se empodera, para actuar en el ámbito de lo colectivo y del mundo de la vida; ello moviliza para la transformación y desplazamiento de las relaciones de poder en la medida en que pueda convertirse o articularse, como movimiento social. El proceso educativo debe permitir que las personas desarrollen su capacidad de interpretar la realidad y de transformarla, haciendo uso creativo del conocimiento construido, los valores y desarrollos, considerando además, la apropiación del acervo cultural propio de su comunidad. 
Esta práctica social y pedagógica desarrolla acciones intencionadas que creen y fortalecen la conciencia social; dista de posturas en las que se asume el conocimiento como un proceso de asimilación que responde a intereses técnicoproductivos centrando su preocupación, en los procesos de enseñanza-aprendizaje. Se acerca mejor, a quienes consideran que el punto de partida del proceso educativo, lo constituyen las representaciones sobre las realidades o los saberes de los participantes, en una dinámica de recontextualización y reorganización para la producción de conocimientos (Ramírez, 2010). Muestra de manera reiterada, una gran preocupación por desarrollar metodologías, herramienta, estrategias, criterios, coherentes con su intención crítica y emancipadora. Por ello acude a principios dialógicos entre los diferentes saberes, al uso de proyectos educativos, comunitarios, la problematización activa y participante de la realidad, la deconstrucción, la negociación cultural, entre otros, como reconocimiento de la acción pedagógica, como práctica liberadora que recoge la diversidad, en tanto pone en cuestión los modelos educativos homogeneizantes.

Como sostiene Ramírez, "el asunto metodológico se recoge bajo la lógica de Acción-Reflexión, Acción-Práctica, Teoría-Práctica, en las que se rescatan las metodologías activas de las pedagogías modernizadoras y los enfoques constructivistas y socializadores de las nuevas pedagogías” (2010).

Reconocemos entonces como cimiente del programa de Educación Integral para Jóvenes y Adultos que la educación popular en el dominio de lo simbólico, permite que los participantes organicen de otra manera las relaciones y representaciones sociales de los problemas que los afectan, tomándolos como contenidos de trabajo, en las prácticas educativas populares. Esas teorías o construcciones interpretativas que los sujetos construyen, deben dar sentido y orientar sus prácticas en diferentes dominios de la vida social. Por ejemplo, debe contribuir, a fortalecer las organizaciones de control público popular y comunitario sobre el Estado, lo que corresponde a su función organizadora.

\section{La alfabetización}

Eso sí, nuestra maldición eterna para aquellos que, para engañarnos y vendernos espejitos, se disfrazan de nosotros y tampoco son los otros. En realidad no son nada, tan solo lacayos de su propio pensamiento colonizado.

La cooperativa que marca

Historia. Experiencia de sistematización, 2003

Una de las prácticas más significativa en el ámbito de la historia de la educación de adultos y la educación popular, sin lugar a dudas, es la alfabetización. Por ello a continuación abordaremos algunos elementos que la fundamentan 
y contextualizan y que retomamos como categoría fundante del programa sistematizado.

En 1985, en la realización de la cuarta conferencia Internacional sobre Educación de Adultos, titula su declaración como el Derecho a Aprender y recoge la condición de derecho fundamental porque a partir de éste "el ser humano deja de ser un objeto a merced de la circunstancia para convertirse en un ente responsable de su propia historia". Indica que el derecho a aprender es: saber leer y escribir, formular preguntas y reflexionar, desarrollar imaginación y creatividad, interpretar el medio circundante y ser protagonista de la historia, tener acceso a los recursos educativos y desarrollar competencia individuales y colectivas. Parte de este proceso es la alfabetización.

Sin embargo, en las condiciones actuales, la alfabetización no es parte de las agendas de los organismos multilaterales y de financiamiento como política para los países de la región; recomiendan que es prioritario volver la mirada hacia la niñez y que los pocos recursos que hay, deben destinarse a ello. Parece que la ineficiencia de los programas desarrollados en este sentido, puede ser una de las razones que se dan para hacer dichas recomendaciones. Estas razones, sin embargo, no son de peso, si consideramos que la educación de los niños y la de los adultos, son inseparables. En la medida en que eduquemos al adulto las condiciones de vida de los niños, serán mejores. No es posible colocarlas en oposición. Además, no hay estudios serios que demuestren que efectivamente las experiencias en alfabetización fracasaron. Existe amplia evidencia que muestra que la educación de los padres y especialmente de las madres, impacta de manera importante en la vida de sus hijos e hijas en cuanto a: salud, nutrición, cuidado, protección, asistencia y permanencia en la escuela, entre otros. En este sentido, el nivel educativo de los adultos-padres, y los usos concretos de la lectura y la escritura en el hogar, estimulan o inhiben el desarrollo de sus hijos. En todas las regiones, países y culturas, las mujeres que no saben leer ni escribir confiesan que una de sus mayores motivaciones para aprender, tiene que ver con la vida escolar de sus hijos: ayudarles a hacer las tareas, sentirse más seguras para acudir a la escuela, asistir a las reuniones de padres de familia o hablar con los profesores. Es tan importante la educación de padres y madres para el bienestar de los niños que algunos educadores creen que el derecho de niños y niñas a la educación básica, debería incluir el derecho a madres y padres educados (Torres, 2006).

Para efectos de la sistematización, reconocemos a la alfabetización como un aprendizaje que le permite a los sujetos aprender a escribir su vida, como testigo de su historia; el método de alfabetización es asumido por Freire como práctica de la libertad; en este sentido, la pedagogía se hace antropología. El método no apunta a la eficiencia técnico-pedagógica. Él en sí mismo es una forma de ver lo pedagógico y la alfabetización, como conciencia, sentido y alcance del humanismo. Es decir es un aprendizaje durante toda la vida. 
El concepto de alfabetización abarca múltiples dimensiones, principalmente la individual y la social. La primera se refiere a la posesión individual de capacidades relacionadas con la lectura y la escritura, que incluyen no sólo la decodificación de palabras, sino a un amplio conjunto de habilidades de comprensión e interpretación como, por ejemplo, establecer relaciones entre ideas, hacer deducciones, reconocer lenguaje figurado, combinar información textual con información extra-textual, etc. Tales habilidades deben ser aplicadas a una amplia gama de textos. La dimensión social de la alfabetización tiene que ver con las prácticas sociales que involucran a la escritura y a la lectura en determinados contextos. Lo que está en juego, en ese ámbito, son los objetivos prácticos de quien utiliza la lectura y la escritura, las interacciones que se establecen entre los que se comunican por medio de ella, las demandas de los grupos sociales, las representaciones y los valores asociados a la lectura y la escritura que un determinado grupo cultural asume y difunde.

Para la práctica alfabetizadora con jóvenes y adultos es fundamental buscar las conexiones entre esas dos dimensiones, ya que el quehacer pedagógico consiste exactamente en la orientación sistemática del desarrollo de los individuos y de su inserción en un contexto sociocultural específico. En el caso de la educación formal en sociedades letradas, el proyecto consiste prioritariamente en la capacitación de los individuos para que con algún nivel de autonomía hagan uso intenso y diversificado de la lengua escrita. Cuando Paulo Freire destaca la importancia de la lectura del mundo, antes que la lectura de la palabra, está llamando la atención hacia esa dimensión extralingüística que es esencial para que el proceso de alfabetización de hecho haga la diferencia para los jóvenes y los adultos. Asi mismo Rosa María Torres sostiene que "La familia y la comunidad local tienen un papel fundamental en hacer de la alfabetización un recurso necesario y gozoso, no sólo en la infancia sino durante toda la vida. Además de acceder a la escuela, es importante tener acceso a actividades culturales y espacios de recreación, a una cancha deportiva, a una biblioteca, a un museo, a un centro informático, a periódicos y otros medios masivos de comunicación, etc. Todo ello contribuye a estimular el aprecio por y el desarrollo de una cultura de aprendizaje permanente" (2006).

Es preciso entonces que, los jóvenes y adultos estén motivados para continuar aprendiendo, que se interesen por lo que se les presenta a futuro, sepan monitorear sus propios procesos de formación, tengan ánimo para modificar sus condiciones de existencia y busquen una vida mejor para ellos y para la comunidad. Los efectos de la alfabetización dependen del contexto psicosocial en el que se realiza.

Desde esta perspectiva, deja de ser fin en sí mismo para convertirse en una manera de preparar a los participantes para desempeñarse social, cívica y económicamente; sobrepasando los límites de la lectura y la escritura en sí. Esta postura de la alfabetización funcional, es decir, ese aprendizaje lecto-escritor, se 
convierte en un pretexto para desarrollar capacidad de leer la realidad, de modo que se logren desarrollos para transformarla, hacer críticas las condiciones de dominación y sujeción de los ciudadanos a los poderes locales o del entorno, preparar para el trabajo y para mejorar las condiciones de vida y recuperar críticamente lo cotidiano. La reflexión sobre las problemáticas de la cotidianeidad, la búsqueda de sus causas y de acciones coherentes para la solución pertinente de las mismas, conforma uno de los propósitos de la capacidad de leer y escribir.

Recuperando el pensamiento de Freire diremos que alfabetizar asume una acción no neutral que implica procesos de reflexión y pensamiento crítico sobre la realidad de lo cotidiano; es un proyecto de lo comunitario y lo colectivo, porque no se aprende solo; se articula con la praxis y depende de los ritmos y procesos individuales. Las acciones alfabetizadoras siempre tienen una finalidad, que surge en el contacto social y que refleja la concepción y proyecto de sociedad de quienes lo formulan. A través de la lectura y la escritura un pueblo se perpetúa. Por eso es esencial en una acción alfabetizadora, incentivar la producción y circulación cultural haciéndola útil y necesaria para el desarrollo de la comunidad. Solo así dejará de ser ajeno al proceso y se convertirá en un elemento de aprendizaje que fortalezca su autoafirmación e identidad cultural. Realizar una acción de este tipo con un interés emancipador supone tener en cuenta: la cultura, oralidad, formas de aprendizaje y motivación del adulto, relación entre pensamiento, palabra y lenguaje, formas de elaboración de material de aprendizaje que recoja elementos culturales de los alfabetizandos, entre otros.

Hemos dicho que esta apuesta pedagógica para la alfabetización, asumida por el programa sujeto de sistematización, supone estrategias, herramientas y metodología que recuperen el diálogo de saberes, la participación, la diversidad y la alteridad como criterios de constitución. En el segmento siguiente hablaremos de estos principios pedagógicos para el modelo de alfabetización.

\section{La pedagogía y las prácticas de la educación popular}

La mirada de lo pedagógico en la educación popular supone articular de manera crítica tres elementos fundamentales: inicialmente articular lo que corresponde a los saberes, representaciones y juicios, sistematizados y acumulados, pertenecientes al orden de las intencionalidades político-éticas otorgadas a las prácticas de educación popular; al orden de las racionalidades metodológicas a partir de las cuales se suscita la movilización y acción social como resultado del proceso educativo; al orden de la crítica de la realidad y la construcción de legitimidad y empoderamiento de quienes participan. En segundo lugar, recoge algunos cuestionamientos que han suscitado posturas críticas según Ramírez, frente a al politicismo, al basismo, al populismo, al didactismo, al metodologismo y muchos otros "ismos" que la condujeron durante algunos años 
a un fuerte aislamiento (2010). Finalmente, la articulación de la conciencia de incompletud, por cuanto es a partir de procesos de diálogo e interacción con otros modelos y escuelas de pensamiento educativo, como logramos avanzar en las construcciones colectivas y en el proceso de descentramiento.

Estas consideraciones, presentan el espacio de lo pedagógico enriquecido por el debate, la superación de lo disciplinar, la recuperación de lo ético y lo político y lo epistémico, convirtiéndolo en un saber, desde la práctica de la educación popular. Es importante precisar que esta educación surge en oposición a la educación domesticadora y bancaria y que planteó unas nuevas relaciones entre quien educa y es educado, el mundo y quiénes aprenden, la toma de conciencia de la propia realidad y la posibilidad de relacionar una realidad histórica y el lenguaje escrito, con fines emancipatorios y transformadores. Supone una propuesta metodológica activa y participativa que recoja las formas, medios y mediaciones pertinentes para la construcción de sentidos y contenidos, desde una perspectiva de fortalecer las organizaciones sociales y los movimientos populares y de gestar una cultura política y ciudadana. Los dispositivos pedagógicos están orientados a intervenir en el mundo de las representaciones de actores populares, a partir del reconocimiento de sus saberes y valores, para reorganizar prácticas y relaciones sociales, en una dinámica de producción de saberes y conocimientos.

La educación entendida entonces como práctica social, se constituye en un ámbito de reflexión e investigación pedagógica. Así esta última en la educación popular toma dos sentidos: como un discurso comprensivo del acto educativo, sus dispositivos y mediadores, sobre las relaciones, sentidos, significaciones, intencionalidades de quienes participan y como reorientadora de las prácticas educativas. Se evidencia así su relación con la dimensión cultura.

Lo pedagógico en la EP cuestiona lo educativo centrado en la enseñanza y propone a la educación como una práctica social articulada a los espacios culturales y de poder que pretende afectar las formas de comprender y de actuar de los participantes; así que recupera el aprendizaje como eje central de la relación pedagógica. En este sentido Ramírez dice que: se centra en las experiencias sociales, prácticas y saberes cotidianos de los individuos y grupos; opera con dispositivos orientados a reestructurar y reorganizar estas experiencias, prácticas y saberes y por tanto, operar en el mundo de la vida cotidiana y de las relaciones sociales; afecta las subjetividades individuales y colectivas; multiplica los espacios y tiempos educativos por efecto de los procesos de socialización; se coloca en la perspectiva de la reconstrucción de una subjetividad histórica con intereses transformadores; asume al sujeto como actor activo de los procesos de aprendizaje y en este sentido, lo entiende como una construcción (2010).

Esta práctica pedagógica recoge principios tales como la: orientación del aprendizaje para la participación; valoración de los saberes populares y ancestrales de cada región; promoción del diálogo y la comunicación; promoción de una experiencia educativa problematizadora, crítica, reflexiva, significativa y 
pertinente; promoción del aprendizaje cooperativo y por último, la promoción de la responsabilidad frente al proceso de aprendizaje de cada uno y de los resultados que obtiene de manera individual así como de los progresos del grupo, para transitar abiertamente hacia la autonomía y la independencia. Estas orientaciones se recogen en la propuesta de alfabetización que desarrollamos en la UNAD.

\section{Metodología}

Entendiendo la sistematización como una práctica investigativa del orden cualitativo en el ámbito social y educativo, reconocemos su aporte además como proceso de evaluación de experiencias en donde la dicotomía sujeto que conoce y sujeto conocido desaparece y el interés por contrastar la teoría y la práctica se fortalecen. En este sentido este trabajo tuvo una pretensión fundamental en torno a donde los diferentes actores construyeran y reflexionaran sobre los sentidos desde sus propios horizontes culturales y sociales.

Para cumplir los objetivos propuestos, se definieron categorías de análisis de la información. Se hizo una revisión documental que incluyó: Secuencia Didáctica, Manual de caracterización de la población, Documento marco sobre competencias, Manual del facilitador, Proyecto de investigación pedagógica, Esquema temático, Cartillas inconclusas, Planeador y Diario de campo.

Así mismo se definió la necesidad de realizar entrevistas a los responsables de la ejecución, coordinación y seguimiento del proyecto, por el CNR y la por la UNAD, por cuanto la sistematización asume procesos de participación de los actores, se definió un trabajo de campo de manera que, se pudieran discutir y analizar con todos ellos, las categorías propuestas; en este sentido se programaron encuentros con los facilitadores, alfabetizados, organizaciones y comunidades participantes y coordinadores locales, en terreno. En este sentido fue necesario hacer acuerdo con los diferentes actores de la experiencia, para definir las rutas, metodologías, tiempos, categorías de análisis y el compromiso institucional. Para ello, se programaron tres salidas a terreno incluyendo las ciudades de Santa Marta, Cúcuta y Bogotá en la localidad de Soacha, respectivamente.

Como resultados de estos acuerdos se definieron las categorías las consideradas fundantes que orientaban el programa. a) Antecedentes: lo diferente; transición de la educación formal a educación popular; justificación; valor agregado; b) Población meta; c) Apuesta por la visibilidad del cambio; d) Metodología: intereses, preguntas, problemas, frases; e) Desarrollo temático como respuesta a caracterización, al mapeo, a la problematización, a lo comunitario; f) Conocimiento popular: tradición educativa, apropiación, aplicación, evaluación, co-evaluación; g) Didáctica: proyecto, frases generadoras, organización del 
tiempo, materiales educativos; h) Aporte e impacto en políticas públicas e i) Concepto de conocimiento.

En cada ciudad se realizaron talleres con los participantes del proceso, aclarando que todos estaban en condición de desplazamiento por cuanto esa fue una de las características del programa desde el inicio, asistiendo a los encuentros pedagógicos con grupos en proceso de alfabetización, así:

Cúcuta: Una reunión con el grupo de adultos que cursaba Ciclo II del programa. Asistentes: 16 personas entre mujeres, y hombres.

Santa Marta: Dos reuniones; una con mujeres cabeza de familia cursando Ciclo II. Asistentes: 18 mujeres y la otra con un grupo de Ciclo I, asistentes 8 personas entre hombres y mujeres.

Soacha: Grupo Ciclo I. Asistentes: 20 personas entre hombres y mujeres.

Además en las visitas a Soacha, Santa Marta y Cúcuta, se llevaron a cabo talleres de reflexión con habitantes de las comunidades, barrios y zonas involucradas, líderes locales, alfabetizadores, alfabetizados y asesores pedagógicos. De igual forma, se sostuvieron reuniones con el Coordinador del programa para el NRC y la Vicerrectora de Desarrollo Regional por la UNAD y los coordinadores en terreno para ambas instituciones.

En las reuniones con los grupos se conversó acerca de las impresiones y apreciaciones del programa, pero teniendo en cuenta indagar por esas categorías fundantes. Se desarrollaron a manera de conversatorios de modo que para los participantes fuera posible expresarse. Finalmente se programó un encuentro con el equipo de trabajo de la UNAD y del CNR.

Tanto la revisión documental como el trabajo de campo, ofrecerían información para el análisis de las categorías, la reflexión y análisis teórico y la oportunidad de aprender de la experiencia y avanzar en su consolidación y mejoramiento. Con dicha información y los registros de las reuniones, conversatorios y talleres se elaboraron matrices para cada categoría lo que permitió la triangulación de la información y el análisis de resultados.

\section{Resultados}

Luego de una revisión inicial de las matrices, encuentros, diálogos y reflexiones colectivas encontramos que: La coordinación central del proyecto ofrece directrices nacionales que en ocasiones, dificultan la recontextualización y apropiación regional. Sin embargo, no existe un documento que integre 
los componentes del proyecto pedagógico, lo que dificulta hacer una lectura articulada del mismo. Esta centralización es clara al hablar de los materiales que se que producen para la formación de los facilitadores a partir del diplomado, pues se trabajan para todas las regiones de la misma manera, al igual que aquellos que se usan en los ejes temáticos y las unidades. El material pedagógico denominado cartillas inconclusas, no evidenció la forma cómo se construye y cómo se usa en las regiones; por información del Coordinador Nacional por la UNAD, en ese momento se estaba revisando, pero no fue posible reconocerlas en el campo. Uno de los coordinadores mencionó también que hicieron las cartillas pero tuvo que enviarlas a revisión a Bogotá. Esto último representa de nuevo una ausencia de autonomía en la elaboración y uso de materiales. Esto claramente desde una propuesta de Educación Popular cuyo propósito es el trabajo autónomo, se constituye en una paradoja.

Por otro lado, se evidencia una tensión entre lo normativo y legal curricularizante y la apuesta de la educación popular y comunitaria, centrada en los principios freirerianos; esto se evidencia en la evaluación, la planeación, la certificación de cursos, entre otros. El uso de pruebas objetivas, exámenes y calificaciones para responder a las exigencias de validación del Ministerio de Educación Nacional representan ejemplos de esa tensión. Otro elemento que emerge es la necesaria dependencia de una institución formal de educación para otorgar los certificados; se pudo precisar que para acceder a estas certificaciones en algunas ocasiones se debía hacer parte de los procesos curriculares y administrativos, de dichas instituciones, aún cuando limitaran la acción del programa de alfabetización.

Los participantes manifestaron satisfacción con el proceso en cuanto a la adquisición de herramientas ciudadanas, de derechos y de participación social; precisaron que los cambios en cuanto a lo comunicativo, las relaciones de convivencia familiar y ciudadana y la exigencia de sus derechos fueron grandes en el tiempo y les permitieron transformar sus realidades.

Frente a las categorías tenemos que en cuanto a los antecedentes: lo diferente; transición de la educación formal a educación popular, justificación y valor agregado, los coordinadores y el equipo central argumentaron tenerlos claros frente a dejar capacidad instalada, el trabajo con organizaciones aliadas, la recuperación de saberes ancestrales y locales, pero en los actores regionales no fue fuerte el sentido de la educación popular; de todas maneras la formación en derechos, se constituye en un valor central de la propuesta. De igual manera, frente a la población meta, el equipo de la UNAD precisó tener claro que la oferta del programa era para todas las personas con interés para alfabetizarse y en condición de desplazamiento, así que no hay un referente de edad sólo que sean jóvenes y adultos; en las visitas se pudo precisar que mucha de esta población la conformaban mujeres y adultos mayores. En este sentido hay ruptura con las apuestas del Consejo Noruego por cuanto su interés giraba en torno a jóvenes y adultos menores de 50 años. Esto generó algunas tensiones interinstitucionales. 
Para la categoría que corresponde a las competencias encontramos que reconocen que las fundamentales son: las ciudadanas, de derechos y de solidaridad. En la práctica lo que pudo verse es que a pesar de tener en cuenta estrategias participativas y que recogían las problemáticas de la población, por un lado, no se contextualizaban porque terminaban siendo para todas las regiones iguales y por otro, se privilegió lo cognitivo: matemáticas, lenguaje, ciencias. Esto se evidenció en la revisión de la secuencia didáctica, los ejes temáticos, los encuentros, los cuadernos, los diarios de campo y sobre todo la evaluación que privilegiaron los avances en lo cognitivo. No se partía de frases problematizadoras o proyectos pedagógicos; estas frases fueron resultados de los encuentros que luego se repetían en planas, desdibujando el sentido del universo vocabular de la apuesta de la educación popular. Se mezclaba claramente, lo tradicional con el interés de la educación de adultos. Los proyectos no fueron transversales porque se hacían al terminar los ejes temáticos como una actividad didáctica complementaria.

Con respecto a la apuesta por la visibilidad del cambio es difícil precisarla; resulta en un híbrido entre lo tradicional y lo popular; al leer los documentos es clara esta condición. Se habla del aprendizaje significativo, del constructivismo y se toca Vigostky en lo pedagógico; si bien es cierto estas son propuestas pedagógicas interesantes no hay fortaleza de la pedagogía popular que sería la apuesta por el cambio. Entre otras, existe un peligro al trabajar con las instituciones educativas: formalizar el programa como alfabetización tradicional.

En palabras de Fernando Granja coordinador de Cúcuta por el CNR: "la alfabetización se volvió el centro y no el pretexto", para la transformación social. En este sentido, es importante articular pedagogos como coordinadores, pero también y fundamentalmente educadores populares. Sabemos que las escuelas normales y los programas de formación de profesores tienen vacíos en la formación especialmente en lo que al interés por el cabio y transformación estructural y formal de la educación se refiere, así que puede ser contraproducente que ellos no tengan elementos para articularse a una propuesta donde lo popular y comunitario, es el centro. Habrá que revisar estos criterios de vinculación.

Este análisis nos introduce en los resultados de la siguiente categoría que perfila el conocimiento popular en tanto tradición educativa, apropiación, aplicación, evaluación, co-evaluación; aquí las inconsistencias son evidentes por cuanto, por ejemplo, a pesar de reconocer el soporte en el saber popular, los materiales no lo evidenciaron. Los materiales de lectura y de trabajo eran recogidos y evaluados desde la coordinación central y lo que se pudo observar es que no se recontextualizaban. El diplomado a través del cual se formaban los formadores en territorio era uno para todas las regiones, al igual que los ejes temáticos, las lecturas y la estructura de los Encuentros. La estructura del diplomado de formación de los facilitadores trabajaba multiformatos, pero desde los asesores nacionales se pedía que la información fuera virtual. No siempre es fácil acceder a estos medios en especial en zonas alejadas de una sede de la UNAD. 
Por otro lado en lo que se refiere a la existencia de las rúbricas, término acuñado para los formatos de registro, control y seguimiento, se constituye en algo que de nuevo, paradójicamente, contradice la educación desde lo comunitario y popular. La acción se tornó muy rígida y no fue el resultado de una construcción colectiva. Se pudo revisar un módulo que opera como cartilla inconclusa, estrategia didáctica que soportaba la propuesta de alfabetización; su densidad y difícil manejo para un alfabetizando y para un facilitador, fueron acentuadas. Además las lecturas y materiales son muy académicos y de alta complejidad. Los encuentros, estrategia pedagógica por esencia se vuelven muy rígidos y pueden terminar siendo clases como en algunos lugares, las denominan los participantes.

En conclusión, es posible afirmar que el proyecto es muy valioso y ofrece herramientas que fortalecen la autonomía de los participantes, el análisis crítico de la realidad, la formación ciudadana y la solidaridad. Vale la pena revisar algunas condiciones de orden pedagógico fundamentalmente pues, la estrategia de intervención caracterizada por las fases de formación a agentes educativos, mapeo, focalización, caracterización, consolidación de equipos locales y la implementación de la alfabetización, junto con el diplomado, se deben mantener y enriquecer. Particularmente el diplomado debe recoger la experiencia para reflexionarla y adecuar los textos, a las condiciones particulares de los entornos donde se lleva a cabo la experiencia.

Es necesario construir un documento que precise los elementos conceptuales, metodológicos y de intervención que caractericen el programa de manera precisa. Muy seguramente han quedado aspectos importantes por reconocer y será necesario trabajar en la construcción de los materiales y el énfasis en la educación popular. Finalmente Es preciso reconocer y así lo manifiestan los participantes que los materiales y documentos son muy ricos en información, orientación y conceptualización pero pueden hacerse más pedagógicos y menos densos. Apoyarse en técnicas narrativas de la educación popular y conservar rigor en su consolidación; su lógica debe ser fácilmente comprendida al igual que su organización.

Este artículo, precisa que a partir del ejercicio de sistematización fue posible formular el Modelo de Alfabetización que se presentó al MEN para su validación.

\section{Discusion}

\section{Aprendizajes de la experiencia}

La educación popular que comprende la educación formal y permanente, en esta experiencia particularmente, se lleva a cabo en escenarios sociales multiculturales, ha ayudado en la construcción, desarrollo de autonomía 
y sentido de responsabilidad al reforzar las capacidades para transformar las relaciones sociales de sus actores. Podemos hacer esta afirmación por cuanto de manera reiterada especialmente las mujeres que participaron en el proceso, relataron experiencias de maltrato y violencia que fueron revertidas; esa posibilidad de exigir derechos logró transformación de la realidad familiar promoviendo coexistencia, tolerancia y participación activa, con lo que descubrieron que es una conquista reflexiva a partir de la que se configuraron en sujetos de su propio destino histórico. Esta ganancia además, según sus relatos, fortaleció la participación activa de estas mujeres en sus propios aprendizajes, en su implicación cualitativa, sobre sus procesos de vida personal y colectiva, además de favor la posibilidad de hacer una lectura crítica de la estructura social y de relaciones de género, de las condiciones de exclusión, desigualdad y de marginalidad, en sus entornos.

Como práctica social pedagógica, la educación popular acude a principios dialógicos entre saberes, al uso de proyectos educativos, la problematización activa y participante de la realidad, la negociación cultural, entre otros, como reconocimiento de la acción pedagógica, como práctica liberadora que recoge la diversidad, en tanto pone en cuestión los modelos educativos homogeneizantes; al mirar y comprender la apuesta del programa desarrollado entre la UNAD y el NRC pese las necesidades encontradas, se hace clara una acción pedagógica que se sustenta en los aspectos mencionados, a través del uso de metodologías participativas, estrategias pedagógicas dialogantes y materiales recontextualizados. En general los alfabetizandos y los alfabetizadores que son de la comunidad también, se refirieron a la experiencia como única y le concedieron la bondad de reconocerlos, fomentar la expresión cultural y del lenguaje local, como fortaleza del programa. Los materiales les significan ya que se construyen sobre sus realidades.

Finalmente, la alfabetización como acción pedagógica de la educación popular asume dos dimensiones especialmente: la que se refiere a la posesión individual de capacidades relacionadas con la lectura y la escritura, incluyendo no sólo la decodificación de palabras, sino a un amplio conjunto de habilidades de comprensión e interpretación, relacionar ideas, deducir, reconocer símbolos, combinar información textual con información intertextual o extra-textual, etc.; y la que reconoce la dimensión social que tiene que ver con las prácticas sociales que involucran a la escritura y a la lectura en determinados contextos. En otras palabras, el uso práctico de quien las utiliza y la interacción con quienes se comunican por medio de ella, las demandas de los grupos sociales, las representaciones y los valores asociados que un determinado grupo cultural asume y difunde. En este sentido esta experiencia ha hecho visible estas dos dimensiones; por un lado quienes participan logran desarrollar sus habilidades lectoras y escriturales de manera práctica, permitiéndoles expresar sus ideas y formas de pensar y por el otro, ofreciéndoles la posibilidad de hacer parte de sus grupos sociales y sus prácticas comunicativas. Escribir un derecho de 
petición, un reclamo a una institución, un discurso para promover derechos, son algunos de los ejemplos que relataron los diferentes actores y que pudieron ser observados en los registros del programa.

\section{Conclusiones}

La sistematización del programa de alfabetización desarrollada por el convenio UNAD-NRC 2008-2010, permitió recoger las impresiones, apreciaciones y experiencias vividas durante los tres años del programa y poner en diálogo a todos los actores.

Los intereses institucionales pueden potenciarse en apuestas que los pongan en acción e interacción a través de acuerdos; tanto la UNAD como el NRC, son organizaciones cuyos propósitos e intereses difieren pero que al revisar el programa, logran articularse y llevar a cabo de manera exitosa y coordinada sus acciones.

Fue claro que las diferentes poblaciones participantes manifestaron haber crecido, aprendido y transformado sus realidades a partir de la experiencia de alfabetización; los alfabetizadores reconocen su contexto claramente y su acción para mejorarlo; los docentes y coordinadores ven expresados sus intencionalidades pedagógicas en estrategias y materiales que proveen elementos de formación pertinente y clara para los y las alfabetizadas; los alfabetizados a su vez, reconocen aprendizajes significativos y mejoramiento en la calidad de sus vidas y entornos familiares, pero también comunitarios.

Finalmente pese a los desencuentros las instituciones del convenio reconocen las bondades, avances y oportunidades del programa. De hecho a partir de esta investigación se valida el Modelo de Alfabetización de la UNAD y se presenta para aprobación ante el Ministerio de Educación Nacional. 


\section{Referencias bibliográficas}

Cendales, L. 2002. Bases teóricas de la sistematización de proyectos económicos. Bogotá: Dimensión Educativa.

Mariño, G. 2008. Módulo de Alfabetización. Bogotá: UNAD.

Quijano, A. 2009. Colonialidad del poder y subjetividad en América Latina. Vicerrectoría de Desarrollo Regional y Proyección comunitaria. Bogotá: Universidad Nacional Abierta y a Distancia. Cátedra Orlando Fals Borda.

Ramírez, J. 2010. Módulo de Educación Popular, sus significados y retos. Bogotá: CINDE.

Rodríguez, E. 1990. Antecedentes históricos de la alfabetización. Alfabetización culturizadora, funcional y liberadora, avance a partir de la experiencia Freireriana. Método Psicosocial, A. Comprometida. Piragua 2, CEAAL.

Torres A. 1999. Ires y venires de la Educación Popular en América Latina. Revista Práctica No 19. Dimensión Educativa. Bogotá: Maestría CINDE.

Torres, A. 2009. Educación popular y paradigmas emancipadores. Revista Latinoamericana de Educación y Política La Piragua. No 30. Panamá.

Torres, R. 2006. Alfabetización y aprendizaje a lo largo de toda la vida. Revista Interamenricana de Educación de Adultos. CREFAL. Disponible en: http://tariacuri. crefal.edu.mx/decisio/d17/sab4-1.php\#inicio.

UNESCO. 2006. La alfabetización un factor vital. Informe de Seguimiento de la EPT en el Mundo. Disponible en: http://unesdoc.unesco.org/images/0014/001442/144270s. pdf

Zaylín, B. 2008. Educación popular, cultura e identidad desde la perspectiva de Paulo Freire. En: Paulo Freire. Contribuciones para la pedagogía. Moacir Godotti, Margarita Victoria Gomez, Jason Mafra, Anderson Fernandes de Alencar (compiladores). CLACSO, Consejo Latinoamericano de Ciencias Sociales, Buenos Aires. Disponible en: http://bibliotecavirtual.clacso.org.ar/ar/libros/campus/freire/06Brito.pdf 